\title{
Réplique de Monsieur Marcel Lafortune au compte rendu de son ouvrage Initiation à la paléographie franco-canadienne. Les écritures des notaires aux XVII-XVIII siècles, par Madame Odette Boulay et Monsieur Jacques Mathieu, paru dans RHAF, volume 37, 3 (décembre 1983) : 464-465.
}

\section{Marcel Lafortune}

Volume 38, numéro 2, automne 1984

Bourgeoisies et Petites Bourgeoisies

URI : https://id.erudit.org/iderudit/304273ar

DOI : https://doi.org/10.7202/304273ar

Aller au sommaire du numéro

Éditeur(s)

Institut d'histoire de l'Amérique française

ISSN

0035-2357 (imprimé)

1492-1383 (numérique)

Découvrir la revue

Citer ce document

Lafortune, M. (1984). Réplique de Monsieur Marcel Lafortune au compte rendu de son ouvrage Initiation à la paléographie franco-canadienne. Les écritures des notaires aux XVII-XVIII siècles, par Madame Odette Boulay et Monsieur Jacques Mathieu, paru dans RHAF, volume 37, 3 (décembre 1983) : 464-465. Revue d'histoire de l'Amérique française, 38(2), 275-276.

https://doi.org/10.7202/304273ar 
Réplique de Monsieur Marcel Lafortune au compte rendu de son ouvrage Initiation à la paléographie franco-canadienne. Les écritures des notaires aux XVII-XVIII ${ }^{e}$ siècles, par Madame Odette Boulay et Monsieur Jacques Mathieu, paru dans $R H A F$, volume 37, 3 (décembre 1983): 464-465.

Il n'est de vérité que dans la profondeur. Autant une vérification rapide et superficielle de notre ouvrage a-t-elle pu aux yeux des auteurs de ce compte rendu soulever de très grandes inquiétudes, autant au départ et de par sa nature elle a porté à une appréciation fautive et erronée. En effet, les inexactitudes et méprises relevées dans cette critique sont nombreuses mais surtout elles étonnent de la part d'historiens, se devant de déchiffrer les sources qui sont le fondement même de leurs recherches; elles commandent et nécessitent les remarques suivantes.

Notre première observation est à l'effet que les actes reproduits en facsimilé n'ont pas tous été dressés par des notaires montréalais; les notaires Romain Becquet et Jean-Baptiste Daguilhe n'ont jamais, à ce que l'on sache, exercé leurs fonctions sur le territoire de Montréal, le premier ayant pratiqué à Québec durant les vingt-trois années de sa carrière, le second à L'Assomption, communément appelé Le Portage, à l'endroit où il tint constamment office jusqu'à la fin de ses jours.

Sur la suppression du «t» au pluriel des adjectifs terminés par «ent» ou «ant» pour y substituer un «S» ou un «Z», et sur l'indistinction graphique entre le «S» et le «f», le «i» et le «j», il faut savoir que c'était là une pratique courante 
à cette époque, qui ne réclamait pas d'explications. Il serait naïf d'attendre de l'auteur, d'entrer au début d'une initiation, dans des détails techniques et de livrer le lecteur à une description d'un type calligraphique déterminé. Notre analyse n'avait pas au départ à poser des critères morphologiques permettant de déceler des modifications dans le sens d'une fixation de certains caractères. En outre, n'ayant pas subi de transformations majeures comme les autres lettres de l'alphabet, les lettres «i», «j» et «o» furent omises dans le tableau de l'évolution de la forme des lettres, qui devait d'ailleurs, se poursuivre dans une publication ultérieure.

En ce qui concerne la remarque sur «Fran» pour François Dollier devenant Jean, elle est juste. C'est par inadvertance que cette erreur s'est glissée jusque chez l'imprimeur; d'autres, minimes et du même genre apparaissent aussi malheureusement, mais elles ne relèvent que de l'imprimerie et feront l'objet d'un errata incessamment. Au reste, le spécialiste le plus expérimenté n'est jamais à l'abri d'une faute de lecture ${ }^{1}$. La transcription des mots «habitant» et «marguiller» est exacte ${ }^{2}$. Chacun sait que l'amputation ou suspension affecte parfois la fin d'un mot, privé de quelques lettres et muni ou non d'un point qui marque l'arrêt du mot. Les auteurs de cette critique n'ont pas tenu compte de la note au lecteur (p. 16 de la brochure), mentionnant que la liste des abréviations ou formes caractéristiques de lettres avait été tirée de différents contrats; il n'était donc pas étonnant de ne pas la retrouver dans le contrat reproduit en fac-similé.

La transcription des textes est conforme aux règles suivies dans le manuel de Maurice Prou, qui a servi d'ouvrage classique de références à des générations d'historiens, de chartistes, de philologues et de médiévistes ${ }^{3}$. Est-il besoin d'ajouter qu'il est universellement notoire en paléographie, que la transcription d'un texte doit le reproduire ne varietur, en autant que son intelligibilité est sauve. C'est là son caractère propre et sa valeur essentielle. En quoi la transcription à la ligne, habituelle en paléographie, est-elle incompréhensible ou «bébête» pour employer un terme indigne d'une critique objective et de bonne foi, à moins de se révéler un primaire en la matière?

Quelle peut être la valeur de la méthode bibliographique enseignée à ses élèves par un professeur quand lui-même éclaire si mal sa lanterne en citant «Lefebvre, J.-J.» et non pas Fernand comme il se doit. Quant à déconseiller l'achat de cette brochure comme étant peu sûre, c'est là une appréciation toute personnelle dont on peut se demander si des lecteurs inexpérimentés dans la lecture des documents anciens n'en jugeraient pas autrement, susceptibles qu'ils sont d'en tirer tout de même quelque utilité.

Société de recherche historique

Archiv-Histo

Montréal

MARCEL LAFORTUNE

\footnotetext{
1 Jacques Stiennon, Paléographie du Moyen-Âge (Paris, 1973), 164.

2 Voir le contrat de Romain Becquet en date du 3 avril 1672, intitulé Déclaration par les marguillers de Notre-Dame de Québec ( $1^{\mathrm{er}} \mathrm{mot}, 16^{\mathrm{e}}$ ligne).

Maurice Prou, Manuel de paléographie latine et française refondu avec la collaboration d'Alain de Bouärd (Paris, $4^{\mathrm{e}}$ édition 1924), in-8. A. de Bourmont, Manuel de paléograplhie des $X V I^{e}, X V I I^{e}$ et XVIII ${ }^{e}$ siècles (Caen, 1881). - Les amis des Archives de l'Ain, Les écritures anciennes. Paléographie française et histoire XVI $-X V I I I^{e}$ siècles (Bourg-en-Bresse, 1982).
} 